\title{
Clinical Findings, Treatments and Outcomes of Transplant Recipients with Metastatic Skin Cancer
}

\author{
Muneeb Ilyas*, Amit Sharma \\ Department of Dermatology, Mayo Clinic Arizona, Arizona, USA \\ "Ilyas.Muneeb@mayo.edu
}

\begin{abstract}
Background: Solid organ transplant recipients develop cutaneous malignancies at a higher rate and with a more aggressive clinical course when compared to the general population. Due to a lack of current information within the transplant literature, we sought to assess the clinical course, treatment and outcomes of metastatic skin cancer in solid organ transplant recipients.
\end{abstract}

Methods: Medical records of 60 solid organ transplant recipients diagnosed with metastatic skin cancer between 2004-2016 were reviewed using the Mayo Clinic Medical Index.

Results: Metastatic skin cancer in solid organ transplant recipients was most commonly due to squamous cell carcinoma (76.7\%), although malignant melanoma (13.3\%) and Merkel cell carcinoma (10.0\%) were also seen. The majority (60.0\%) of patients were immunosuppressed with mycophenolate mofetil, tacrolimus and prednisone. Primary tumors were most commonly treated using excision (35.0\%). Relapsing primary skin cancers were seen in $26.1 \%$ of patients with squamous cell carcinoma, $50.0 \%$ of patients with Merkel cell carcinoma, and $37.5 \%$ in patients with malignant melanoma. Mortality due to disease was highest in patients with malignant melanoma (50.0\%), followed by squamous cell carcinoma (28.3\%), and Merkel cell carcinoma (16.7\%).

Limitations: Due to the retrospective nature of our study, we could not determine the efficacy of treatments for metastatic disease as they were non-random.

Conclusion: Metastatic skin cancer in solid organ transplant recipients has a poor prognosis. Solid organ transplant recipient care providers should initiate early and aggressive treatment of skin cancer, regardless of tumor type, to reduce the risk of developing this serious complication.

Keywords: Skin Cancer, Metastatic Disease, Squamous Cell Carcinoma

Abbreviations: BCC: basal cell carcinoma, HPV: human papilloma, MCC: Merkel cell carcinoma, MM: malignant melanoma, SCC: Squamous cell carcinoma, SOTR: Solid organ transplant recipient.

\section{INTRODUCTION}

Cutaneous malignancy in solid organ transplant recipients (SOTRs) occurs more frequently and with a more aggressive clinical course when compared to those in the general population. Skin cancers account for $40 \%$ of malignancies seen in SOTRs and the majority are squamous cell carcinomas (SCC) and basal cell carcinomas (BCC $)^{1-4}$. Unlike the non-immunosuppressed population, SOTRs are more likely to develop SCC than BCC. A study ${ }^{5}$ in heart transplant recipients found that $89 \%$ of skin cancers in this population were SCC, while $11 \%$ were BCC. The prevailing theory for skin cancer development in SOTRs is that due to diminished immune surveillance, an enhancement of UVinduced DNA damage allows atypical cells to survive and proliferate ${ }^{6,7}$. This theory is supported by Ducloux et al. ${ }^{8}$, who discovered that renal transplant recipients with skin cancers had significantly lower mean CD4+ T-cell counts than those without skin cancer. Moreover, SOTRs are seven-times more likely to develop non-melanoma skin cancer when compared to AIDS patients ${ }^{9}$. This finding suggests that immunosuppression is not a sufficient explanation for skin cancer development in SOTRs. The contribution of carcinogenic immunosuppressants and oncogenic viral infections are also thought to contribute to skin cancer development ${ }^{6}$. SOTRs are at increased risks of human papillomavirus (HPV) and although an association between HPV and SCC has been described, ongoing research suggests that the link is not as clear ${ }^{10}$. 
In comparing SOTRs to the general population, the incidence of malignant melanoma (MM) is twofold and that of Merkel cell carcinoma (MCC) is increased 23 -fold ${ }^{112}$. Cutaneous malignancies in SOTRs metastasize more frequently and cause higher mortality than skin cancers in the general population ${ }^{12-15}$. Melanoma in SOTRs has increased morbidity and mortality compared to immunocompetent individuals ${ }^{12}$. With only $14 \%$ of SOTRs being referred to dermatologists posttransplant, skin cancer severity in SOTRs is underestimated. The primary reason cited for a physician to avoid a dermatology referral was a lack of sufficient medical evidence warranting screening in SOTRs ${ }^{16}$. We conducted this retrospective review in order to update the transplant literature on metastatic cutaneous malignancies in transplant recipients. Due to advancements in medical knowledge and drug therapy, this study provides SOTR care providers with current information on the clinical course, treatment and outcomes of metastatic skin cancers in SOTRs.

\section{MeThODS AND DEFINITIONS}

This study was approved by the institutional review board at the Mayo Clinic. Using the Mayo Clinic Medical Index, we identified 60 SOTRs diagnosed with metastatic skin cancer between 2004-2016. The patients were all on immunosuppressive therapy at the time of their primary skin tumor diagnosis. Patient data collected included demographics, transplant history, immunosuppression, primary tumor and treatment, metastatic disease and treatment, and clinical course.

We defined primary tumor as the primary cutaneous tumor that metastasized. Due to multiple tumors at the site of the primary, a subset of patients had many possible primary cutaneous tumors; therefore, the primary tumor and its treatment were considered unknown within these patients. Metastatic tumors were defined as evidence of disease that is noncontiguous with the primary tumor. Metastatic disease consisted of nodal, non-nodal, and widespread disease. Nodal metastasis described spread to regional lymph nodes. Non-nodal metastasis described spread to other cutaneous sites or organs. Widespread disease involved the presence of metastatic nodal and multi-organ disease. For the purpose of statistical analysis, metastatic disease sites were separated based on tumor type. Metastases from SCC were separated into two groups, internal metastases, which described spread of disease to lymph nodes or internal organs, and cutaneous or in-transit metastases. Patients with nodal and nonnodal metastasis were recorded as having both. Recurrence described regrowth of the primary tumor within the originally treated site. Descriptive statistics were performed.

\section{RESULTS}

The demographics and transplant details of our patients are summarized in Table 1. Our patient population was $96.7 \%$ white, $80 \%$ male, with an average age of 50 years. The kidney was the most common organ transplanted $(80 \%)$, followed by the liver (13.3\%). The most common immunosuppressive regimen in our patients was mycophenolate mofetil, tacrolimus, and prednisone $(60 \%)$.

Table1. Patient Demographics and Clinical Data

\begin{tabular}{|l|l|}
\hline \multicolumn{2}{|l|}{ Number $(\%)$ of patients $(\mathbf{N}=60)$} \\
\hline Demographics & $48(80.0 \%)$ \\
\hline Male & $12(20.0 \%)$ \\
\hline Female & $50 \pm 15.9$ \\
\hline Average \pm standard deviation of age at transplant & $58(96.7 \%)$ \\
\hline White & $2(3.3 \%)$ \\
\hline Other & \\
\hline Organ transplanted & $48(80.0 \%)$ \\
\hline Kidney & $8(13.3 \%)$ \\
\hline Liver & $1(1.7 \%)$ \\
\hline Lung & $1(1.7 \%)$ \\
\hline Pancreas & $1(1.7 \%)$ \\
\hline Kidney/Liver co-transplant & $1(1.7 \%)$ \\
\hline Kidney/Pancreas co-transplant & $7(11.7 \%)$ \\
\hline Predominant Immunosuppressive regimen & $6(10.0 \%)$ \\
\hline Tacrolimus and prednisone & $3(5.0 \%)$ \\
\hline Azathioprine and prednisone & $2(3.3 \%)$ \\
\hline Cyclosporine and prednisone & \\
\hline Sirolimus and prednisone & \\
\hline
\end{tabular}


Clinical Findings, Treatments and Outcomes of Transplant Recipients with Metastatic Skin Cancer

\begin{tabular}{|l|l|}
\hline MM, tacrolimus and prednisone & $36(60.0 \%)$ \\
\hline MM, sirolimus and prednisone & $3(5.0 \%)$ \\
\hline MM, cyclosporine and prednisone & $2(3.3 \%)$ \\
\hline Azathioprine, tacrolimus and prednisone & $1(1.7 \%)$ \\
\hline
\end{tabular}

MM: mycophenolate mofetil.

Primary tumor characteristics and treatment details are summarized in Table 2. 46 (76.7\%) patients had SCC, $8(13.3 \%)$ had MM and $6(10 \%)$ had MCC. A subset of 29 SOTRs who developed metastatic skin cancer did not have an identifiable primary tumor; therefore, data regarding primary tumor site and its treatment was unavailable. The most common known primary sites were the temple (11.7\%), cheek (10.0\%), and scalp (8.3\%). Treatment options included excision (35.0\%), excision and radiation $(8.3 \%)$, Mohs surgery $(5.0 \%)$, radiation only (1.7\%), and amputation $(1.7 \%)$.

Table2. Primary Tumor Characteristics and Initial Treatment Details

\begin{tabular}{|c|c|}
\hline Characteristic & Number $(\%)$ of tumors $(\mathrm{N}=60)$ \\
\hline \multicolumn{2}{|l|}{ Tumor type } \\
\hline Squamous cell carcinoma & $46(76.7 \%)$ \\
\hline Melanoma & $8(13.3 \%)$ \\
\hline Merkel cell carcinoma & $6(10.0 \%)$ \\
\hline \multicolumn{2}{|l|}{ Primary Tumor Site } \\
\hline Unknown $^{\mathrm{a}}$ & $29(48.3 \%)$ \\
\hline Temple & $7(11.7 \%)$ \\
\hline Cheek & $6(10.0 \%)$ \\
\hline Scalp & $5(8.3 \%)$ \\
\hline Trunk & $4(6.7 \%)$ \\
\hline Upper extremity & $4(6.7 \%)$ \\
\hline Lower extremity & $2(3.3 \%)$ \\
\hline Eyelid & $1(1.7 \%)$ \\
\hline Ear & $1(1.7 \%)$ \\
\hline Penis & $1(1.7 \%)$ \\
\hline \multicolumn{2}{|l|}{ Treatment } \\
\hline Unknown $^{\mathrm{a}}$ & $29(48.3 \%)$ \\
\hline Excision & $21(35.0 \%)$ \\
\hline Mohs & $3(5.0 \%)$ \\
\hline Radiation & $1(1.7 \%)$ \\
\hline Amputation & $1(1.7 \%)$ \\
\hline Excision and radiation & $5(8.3 \%)$ \\
\hline
\end{tabular}

${ }^{a}$ Twenty- nine patients had an unknown primary tumor site, most commonly due to multiple possible primary tumors. Thus, treatment details are also unknown.

Table3. Differentiation of Primary Squamous Cell Carcinomas

\begin{tabular}{|l|l|}
\hline Differentiation & Number $(\%)$ of tumors $(\mathbf{N}=\mathbf{4 6})$ \\
\hline Unknown $^{\text {a }}$ & $29(63.0 \%)$ \\
\hline Moderately differentiated & $8(17.4 \%)$ \\
\hline Well-differentiated & $4(8.7 \%)$ \\
\hline Poorly differentiated with perineural invasion & $4(8.7 \%)$ \\
\hline Sarcomatoid with spindle formation & $1(2.2 \%)$ \\
\hline
\end{tabular}

aTwenty-nine of forty-six squamous cell carcinomas had an unknown primary site, most commonly due to multiple possible primary tumors.

Table4. Categorizing Primary Melanomas Based on Breslow's Depth.

\begin{tabular}{|l|l|}
\hline Depth & Number $(\%)$ of tumors $(\mathbf{N}=8)$ \\
\hline Thin $^{\mathrm{a}}$ & $1(12.5 \%)$ \\
\hline Intermediate $^{\mathrm{b}}$ & $4(50.0 \%)$ \\
\hline Thick $^{\mathrm{c}}$ & $2(25.0 \%)$ \\
\hline Unknown & $1(12.5 \%)$ \\
\hline
\end{tabular}

${ }^{a}$ Tumors less than 1mm in Breslow's depth; btumors between 1-4mm in Breslow's depth; ctumors greater than $4 m m$ in Breslow's dept. 


\section{Muneeb Ilyas and Amit Sharma}

The primary tumors within our patient population were further categorized based on their histologic features. $46(76.7 \%)$ of our patients had metastatic SCC and features of the associated primary tumors are summarized in Table 3. Twenty-nine (63\%) metastatic SCCs had unknown primary tumors. Of the remaining primary SCCs, $8(17.4 \%)$ were moderately differentiated, 4 (8.7\%) were welldifferentiated, $4(8.7 \%)$ were poorly differentiated with perineural invasion and $1(2.2 \%)$ was sarcomatoid with spindle formation. We categorized our primary melanomas based on Breslow's depth and these results are summarized in Table 4. Tumors with less than $1 \mathrm{~mm}$ of invasion were categorized as thin, between 1-4mm in Breslow's depth were categorized as intermediate, and greater than $4 \mathrm{~mm}$ of Breslow's depth were categorized as thick. There was no data regarding Breslow's depth for 1 of the primary melanomas. $4(50 \%)$ of primary tumors were intermediate depth, while $2(25 \%)$ were thick and $1(12.5 \%)$ was thin. There was no analysis of histology for primary MCCs as this information was unavailable.

Table 5. Characteristics and Sites of Metastasis for Squamous Cell Carcinomas

\begin{tabular}{|l|l|}
\hline $\begin{array}{l}\text { Feature } \\
\text { Nodal disease }\end{array}$ \\
\hline Nodal metastasis & $20(43.5 \%)$ \\
\hline Non-nodal metastasis & $26(56.6 \%)$ \\
\hline Non-nodal sites & \\
\hline In-transit or cutaneous metastasis & \\
\hline Cutaneous & $29(63.0 \%)$ \\
\hline Internal metastasis & $6(13.0 \%)$ \\
\hline Parotid gland & $4(8.7 \%)$ \\
\hline Internal ear & $5(10.9 \%)$ \\
\hline Lung & $3(6.5 \%)$ \\
\hline Bone & $4(8.7 \%)$ \\
\hline Widespread disease & $5(10.9 \%)$ \\
\hline Nodal disease only &
\end{tabular}

${ }^{a}$ Some patients had metastases affecting nodal and non-nodal sites. Additionally, some non-nodal metastases fell under multiple categories.

Characteristics and sites of metastatic SCCs are summarized in Table 5. 20 (43.5\%) patients had nodal metastasis of their SCC. Of the patients with non-nodal SCC metastases, cutaneous or in-transit metastases were seen in $29(63.0 \%)$ patients, internal metastasis were seen in $18(39.1 \%)$, widespread disease was identified in $4(8.7 \%)$ patients and $5(10.9 \%)$ patients had nodal disease without extranodal manifestations.

Table 6. Characteristics and Sites of Metastasis for Merkel Cell Carcinomas

\begin{tabular}{|l|l|}
\hline $\begin{array}{l}\text { Feature } \\
\text { Nodal disease }^{\mathrm{a}}\end{array}$ \\
\hline Nodal metastasis & $5(83.3 \%)$ \\
\hline Non-nodal metastasis & $1(16.7 \%)$ \\
\hline Non-nodal sites & \\
\hline Cutaneous & $1(16.7 \%)$ \\
\hline Widespread disease & $2(33.3 \%)$ \\
\hline None & $3(50.0 \%)$ \\
\hline
\end{tabular}

${ }^{a}$ Some patients had metastases affecting nodal and non-nodal sites.

Table 6 summarizes characteristics and sites of metastatic MCC in our patients. There was evidence of nodal disease in $5(83.3 \%)$ of our patients, with $3(50 \%)$ of these patients lacking extra-nodal disease. One patient had non-nodal disease without evidence of nodal infiltration. Widespread disease was noted in $2(33.3 \%)$ patients and cutaneous metastasis was seen in $1(16.7 \%)$.

Table7. Characteristics and Sites of Metastasis for Melanomas

\begin{tabular}{|l|l|}
\hline Feature & Number $(\%)$ of Tumors $(\mathbf{N}=8)$ \\
\hline Nodal disease $^{\mathrm{a}}$ & $7(87.5 \%)$ \\
\hline Nodal metastasis & $1(12.5 \%)$ \\
\hline Non-nodal metastasis & \\
\hline Non-nodal sites $^{\mathrm{a}}$ & \\
\hline
\end{tabular}


Clinical Findings, Treatments and Outcomes of Transplant Recipients with Metastatic Skin Cancer

\begin{tabular}{|l|l|}
\hline Cutaneous & $4(50.0 \%)$ \\
\hline Lung & $2(25.0 \%)$ \\
\hline Liver & $3(37.5 \%)$ \\
\hline Spleen & $1(12.5 \%)$ \\
\hline Widespread disease & $3(37.5 \%)$ \\
\hline None & $3(37.5 \%)$ \\
\hline
\end{tabular}

${ }^{a}$ Some patients had metastases affecting nodal and non-nodal sites. Additionally, some non-nodal metastases fell under multiple categories.

Data regarding characteristics of MM in our SOTRs is presented in Table 7. For our MM, nodal disease was evident in 7 (87.5\%) SOTRs. Metastasis to the skin was the most common site (50\%), though spread to the lung (25\%), liver (37.5\%) and spleen (12.5\%) was also seen. Widespread disease was evident in $3(37.5 \%)$ of patients.

Table8. Treatments Used for Metastatic Disease

\begin{tabular}{|l|l|}
\hline Treatment & Number (\%) of Tumors \\
\hline Squamous Cell Carcinoma (N=46) & $18(39.1 \%)$ \\
\hline In-transit or cutaneous metastasis & $4(8.7 \%)$ \\
\hline Excision & $2(4.3 \%)$ \\
\hline Mohs & $2(4.3 \%)$ \\
\hline Excision and Mohs & $1(2.2 \%)$ \\
\hline Internal metastasis & $12(26.1 \%)$ \\
\hline Radiation & $1(2.2 \%)$ \\
\hline Chemotherapy & $2(4.3 \%)$ \\
\hline Excision and radiation & $2(4.3 \%)$ \\
\hline Chemotherapy and radiation & $1(2.2 \%)$ \\
\hline Mohs and radiation & $1(2.2 \%)$ \\
\hline Excision, radiation and chemotherapy & \\
\hline Excision, Mohs and radiation & $3(50.0 \%)$ \\
\hline Unknown & $1(16.7 \%)$ \\
\hline Merkel Cell Carcinoma $(\mathbf{N}=6)$ & $2(33.3 \%)$ \\
\hline Excision and radiation & \\
\hline Radiation & $2(25.0 \%)$ \\
\hline None & $2(25.0 \%)$ \\
\hline Melanoma (N=8) & $1(12.5 \%)$ \\
\hline Excision & $1(12.5 \%)$ \\
\hline Radiation & $1(12.5 \%)$ \\
\hline Excision and radiation & $1(12.5 \%)$ \\
\hline Excision and chemotherapy & \\
\hline Excision, radiation and chemotherapy & \\
\hline None & \\
\hline
\end{tabular}

We separated treatments utilized for metastatic disease based on tumor type as summarized on Table8. For metastatic SCC, we further divided treatment depending on internal versus in-transit or cutaneous metastases. The most frequent treatments included excision $(39.1 \%)$, excision and radiation $(26.1 \%)$ and Mohs surgery $(8.7 \%)$. Additional therapies included chemotherapy (2.2\%), radiation $(4.3 \%)$ and combinations of various treatments. Of the 6 patients with metastatic MCC, $3(50 \%)$ were treated with excision and radiation, $1(16.7 \%)$ with radiation only and $2(33.3 \%)$ patients received no treatment due to severe widespread disease. MM patients were treated with excision (25\%), radiation (25\%), excision and chemotherapy (12.5\%), and other combination therapies. Severe widespread MM treated with palliative care alone was provided for 1 (12.5\%) patient.

Table9. Relapsing Primary Skin Cancers

\begin{tabular}{|l|l|}
\hline Relapse & Number $(\%)$ of Tumors \\
\hline Squamous Cell Carcinoma (N=46) & $12(26.1 \%)$ \\
\hline Yes & $34(73.9 \%)$ \\
\hline No & $3(50.0 \%)$ \\
\hline Merkel Cell Carcinoma (N=6)
\end{tabular}




\begin{tabular}{|l|l||}
\hline No & $3(50.0 \%)$ \\
\hline Melanoma (N=8) & $3(37.5 \%)$ \\
\hline Yes & $5(62.5 \%)$ \\
\hline No
\end{tabular}

Relapse of primary tumors within or adjacent to the treatment scar was found in all skin cancer types. Data on relapse was reported based on cancer type and is presented on Table 9. Of the 46 patients who developed metastatic SCC, $12(26.1 \%)$ had evidence of relapse. This value may underrepresent the actual rate of relapse as the primary tumor was unknown in 29 patients with metastatic SCC. Metastatic MCC relapsed in 50\% of our cases. The rate of relapse was $37.5 \%$ in MM patients.

Table 10. Patient Outcome.

\begin{tabular}{|l|l|}
\hline Outcome & Number $(\%)$ of Tumors \\
\hline Squamous Cell Carcinoma (N=46) & $5(28.3 \%)(\mathrm{N}=18)$ \\
\hline Death due to internal metastasis & $13(28.2 \%)$ \\
\hline Death unrelated to skin cancer & $28(60.9 \%)$ \\
\hline Alive & $1(16.7 \%)$ \\
\hline Merkel Cell Carcinoma (N=6) & $2(33.3 \%)$ \\
\hline Death due to Merkel cell carcinoma & $3(50.0 \%)$ \\
\hline Death unrelated to skin cancer & \\
\hline Alive & $4(50.0 \%)$ \\
\hline Melanoma (N=8) & $1(12.5 \%)$ \\
\hline Death due to melanoma & $3(37.5 \%)$ \\
\hline Death unrelated to skin cancer &
\end{tabular}

Overall outcome in our population is documented on Table 10. 28 (60.9\%) SOTRs with metastatic SCC are alive at the time of data collection. Death due to internal metastatic SCC was seen in 5 $(27.8 \%)$ patients. Only patients with internal metastases were considered when calculating the percent mortality associated with metastatic SCC. $13(28.3 \%)$ patients suffered an unrelated death. Of our SOTRs with metastatic MCC, 3 (50\%) are alive at the time of data collection. Two (33.3\%) patients expired due to unrelated reasons and $1(16.7 \%)$ patient died due to metastatic MCC. Within our population of patients with MM, $3(37.5 \%)$ are alive, 1 (12.5\%) died due to unrelated causes and 4 (50\%) died due to MM.

\section{DISCUSSION}

Cutaneous malignancies in SOTRs have increased incidence, severity, and likelihood to metastasize versus the immunocompetent population ${ }^{12-15,17}$. SOTR care providers are advised to provide early treatment with margin control to cutaneous malignancies ${ }^{3}$. The results of this study provide insight into relevant findings, treatments and outcomes of SOTRs who developed metastatic skin cancer.

SCC, MCC, and MM were reviewed in this study. The number of primary tumors and extent of metastatic disease varied greatly between patients and skin cancer type. SCC was the most common metastatic skin cancer in our patients, which is consistent with the literature ${ }^{5}$. The primary SCCs in our population were mostly moderately or poorly-differentiated with perineural invasion, suggesting an aggressive carcinoma. Additionally, our MM cases had predominantly intermediate and thick primary melanomas. Characteristics of aggressive primary skin cancers in our population are consistent with studies that found similar findings in SOTRs ${ }^{18}$. Farasat et al. ${ }^{19}$ concluded that up to $20 \%$ of high-risk SCCs in SOTRs may metastasize. SCCs were characterized as high-risk if they were poorly-differentiated or had perineural invasion, both of which were seen in our population ${ }^{20}$. Furthermore, Song et al. ${ }^{21}$ found that SOTRs had 2.74 times higher odds of developing non-melanoma skin cancers with aggressive subclinical extension when compared to non-SOTRs. The most common locations for primary skin cancers were on the scalp and face; though, tumors on the trunk, extremities and genitalia were noted. This finding is consistent with reports suggesting facial skin cancer is more aggressive than skin cancer on the trunk or extremities. SCC on the lip, cheek, and preauricular areas is reportedly more aggressive, with a higher risk of metastasis, when compared to lower-leg $\mathrm{SCC}^{22}$. Additionally, Hoersch et al. ${ }^{23}$ report higher local recurrence of invasive melanoma on the face when compared to the trunk or extremities. Primary tumors were mostly treated with excision, radiation, or both. The rates of relapse in our patients varied based on tumor type and it is unclear if any treatments had a measurable effect on tumor relapse or metastasis. 
Regardless of skin cancer type, metastatic disease most commonly spread to regional nodes, as seen within the immunocompetent population. This finding reinforces the need to perform a thorough lymph node exam in SOTRs with skin cancer history ${ }^{24}$. Much like in the general population, the most common extra-nodal site of metastasis in SOTRs was the skin, particularly of the scalp and face ${ }^{24}$. Relapse of primary tumors was evident with all skin cancer types. The number of relapsing SCCs may be underrepresented in our data as the primary site was unknown in some SCC patients. It is thus imperative for SOTR care providers to appropriately treat primary skin cancers with methods that provide details on margins as to decrease the burden of relapsing disease. Due to the retrospective nature of our study and non-randomization of treatments, we were unable to deduce which treatments were superior for managing metastases. The most frequently used treatments for metastatic disease were excision, radiation, or both.

In our population, mortality rates due to skin cancer were lower than previous estimates. In a population of SOTRs, Lloyd et al. ${ }^{16}$ found that $49 \%$ of patients with metastatic cutaneous malignancies died from their disease. Yet, $72 \%$ of these deceased SOTRs were seen by physicians who seldom referred patients for dermatologic screening ${ }^{16}$. Additionally, Ong et al. ${ }^{25}$ found that $27 \%$ of heart transplant recipients died due to untreated skin cancer within four years of their transplant date. These findings highlight the importance of dermatologic screening and early treatment of skin cancers in SOTRs.

Mortality due to metastatic skin cancer varied based on tumor type. Mortality was highest in patients with MM, which is expected based on prior studies ${ }^{26}$. However, mortality associated with metastatic SCC was $28.3 \%$, similar to previous estimates ${ }^{27,28}$. Rashtak et al. ${ }^{28}$ and Harwood et al. ${ }^{27}$ reported death due to metastatic SCC in $9 \%$ and $64 \%$ of their SOTR populations, respectively. Our findings suggest a higher cause-mortality associated with non-squamous cell skin cancers. We believe that improved medical management and changes in immunosuppression may have contributed to the outcomes seen in our population. Over time, advancements in drug therapy have led to novel immunotherapy options for SOTRs. In the past, immunosuppression in SOTRs was predominantly maintained using cyclosporine and azathioprine ${ }^{24}$. A systematic review involving twenty-seven studies found a $56 \%$ increased risk of SCC development in SOTRs on azathioprine versus other immunosuppressants ${ }^{29}$. This is thought to be mediated by azathioprine induced skin hypersensitivity to UVA radiation and increased 6-thioguanine levels in DNA; both of which are thought to increase reactive oxygen species and subsequent SCC risk ${ }^{30,31}$. Sugie et al. ${ }^{32}$ discovered that cyclosporine interferes with p53 signaling and nucleotide excision repair, which may lead to increased SCC development. Cyclosporine is associated with SCC development in a dose dependent manner, and combination with azathioprine and prednisone is associated with a 3 -fold higher risk of skin cancer versus azathioprine and prednisone alone $\mathrm{e}^{33-35}$. Although tacrolimus is a calcineurin inhibitor, the risk of SCC is not as high as with cyclosporine ${ }^{36}$. The most frequently used immunosuppressive regimen in our patients was a combination of mycophenolate mofetil, tacrolimus and prednisone. We feel the change in immunomodulation from agents such as azathioprine and cyclosporine to mycophenolate mofetil and tacrolimus in our population likely contributed to the lower rates of SCC development and subsequent mortality when compared to older studies ${ }^{24,37}$. Studies on another sub-type of immunosuppressant, mTOR inhibitors, have shown beneficial effects in SOTRs with non-melanoma skin cancers. When immunosuppressed patients were switched from calcineurin inhibitors to mTOR inhibitors, Alberú et al. ${ }^{38}$ found that skin cancer rates were three times lower in the mTOR inhibitor group after two years. Alter et al. ${ }^{39}$ reported a significant decrease of SCC development in SOTRs as early as the first year after changing immunosuppression to mTOR-inhibitors. Furthermore, $\mathrm{Gu}$ et al. $^{40}$ reported that sirolimus has an anti-tumor effect and sirolimus-based immunosuppression decreases rates of both SCC and BCC in kidney transplant recipients. A contradictory study ${ }^{28}$ found that sirolimus did not decrease skin cancer in lung transplant recipients; however, this finding may be attributed to the multiple immunosuppressants used in lung transplant recipients. Switching immunosuppression to mTOR inhibitors is an option for SOTR care providers to consider in patients with non-melanoma skin cancers. The development of novel immunotherapy agents, such as ipilimumab (a cytotoxic Tlymphocyte associated antigen 4 inhibitor) and pembrolizumab and nivolumab (programmed cell death protein 1 inhibitors) have heralded a revolution in melanoma treatment and cancer care in general. Yet, these agents may be disallowable in SOTRs given the potential of organ rejection. 
However, Lipson et al. $^{41}$ and Qin and Salama ${ }^{42}$ reported successful management of MM using ipilimumab in two kidney and a heart transplant recipient, respectively. Although no large study on ipilimumab use in SOTRs has been performed, these cases suggest that ipilimumab may be an option for SOTRs with MM. Lastly, Dantal et al. ${ }^{33}$ suggest that lowering immunosuppression in SOTRs may decrease the incidence of cutaneous malignancy and this option may be considered by care providers when dealing with these cases. Changes in immunomodulation and advancements in medical management may play a considerable role in patient outcome. At this time, there is no strong evidence as to guide us when and by what amount immunosuppression may need to be decreased in patients with metastatic cutaneous malignancies.

Our study is limited in its retrospective nature. Due to this, we could not determine the efficacy of treatments as they were non-random. Randomized-controlled trials of treatment options for SOTRs with metastatic cutaneous malignancies are largely absent. As newer targeted agents are developed for cutaneous and other malignancies, there should be an emphasis on using them in affected SOTRs. Given the limited options we currently have, it is essential that SOTRs with skin cancer history should regularly visit their dermatologist for skin and lymph node examinations.

\section{REFERENCES}

[1] Chockalingam, R., Downing, C. \& Tyring, S. K. Cutaneous Squamous Cell Carcinomas in Organ Transplant Recipients. J Clin Med 4, 1229-1239, (2015).

[2] Ulrich, C., Kanitakis, J., Stockfleth, E. \& Euvrard, S. Skin cancer in organ transplant recipients-where do we stand today? Am J Transplant 8, 2192-2198, (2008).

[3] Euvrard, S., Kanitakis, J. \& Claudy, A. Skin cancers after organ transplantation. N Engl J Med 348, 1681-1691, (2003).

[4] Eisemann, N. et al. Non-melanoma skin cancer incidence and impact of skin cancer screening on incidence. J Invest Dermatol 134, 43-50, (2014).

[5] Brewer, J. D. et al. Incidence of and risk factors for skin cancer after heart transplant. Arch Dermatol 145, 1391-1396, (2009).

[6] Athar, M., Walsh, S. B., Kopelovich, L. \& Elmets, C. A. Pathogenesis of nonmelanoma skin cancers in organ transplant recipients. Arch Biochem Biophys 508, 159-163, (2011).

[7] Ponticelli, C., Cucchiari, D. \& Bencini, P. Skin cancer in kidney transplant recipients. J Nephrol 27, 385-394, (2014).

[8] Ducloux, D. et al. CD4 lymphocytopenia in long-term renal transplant recipients. Transplant Proc 30, 2859-2860 (1998).

[9] Grulich, A. E., van Leeuwen, M. T., Falster, M. O. \& Vajdic, C. M. Incidence of cancers in people with HIV/AIDS compared with immunosuppressed transplant recipients: a meta-analysis. Lancet 370, 59-67, (2007).

[10] Dimon, M. T. et al. No evidence for integrated viral DNA in the genome sequence of cutaneous squamous cell carcinoma. J Invest Dermatol 134, 2055-2057, (2014).

[11] Clarke, C. A. et al. Risk of merkel cell carcinoma after solid organ transplantation. J Natl Cancer Inst 107, (2015).

[12] Robbins, H. A. et al. Melanoma Risk and Survival among Organ Transplant Recipients. J Invest Dermatol 135, 2657-2665, (2015).

[13] Barrett, W. L., First, M. R., Aron, B. S. \& Penn, I. Clinical course of malignancies in renal transplant recipients. Cancer 72, 2186-2189 (1993).

[14] Krynitz, B., Rozell, B. L., Lyth, J., Smedby, K. E. \& Lindelof, B. Cutaneous malignant melanoma in the Swedish organ transplantation cohort: A study of clinicopathological characteristics and mortality. J Am Acad Dermatol 73, 106-113 e102, (2015).

[15] Otley, C. C. \& Pittelkow, M. R. Skin cancer in liver transplant recipients. Liver Transpl 6, 253 $262,(2000)$. 
[16] Lloyd, A., Klintmalm, G., Qin, H. \& Menter, A. Skin cancer evaluation in transplant patients: a physician opinion survey with recommendations. Clin Transplant 29, 110-117, (2015).

[17] Brantsch, K. D. et al. Analysis of risk factors determining prognosis of cutaneous squamous-cell carcinoma: a prospective study. Lancet Oncol 9, 713-720, (2008).

[18] Cherpelis, B. S., Marcusen, C. \& Lang, P. G. Prognostic factors for metastasis in squamous cell carcinoma of the skin. Dermatol Surg 28, 268-273 (2002).

[19] Farasat, S. et al. A new American Joint Committee on Cancer staging system for cutaneous squamous cell carcinoma: creation and rationale for inclusion of tumor (T) characteristics. J Am Acad Dermatol 64, 1051-1059, (2011).

[20] Brunner, M., Veness, M. J., Ch'ng, S., Elliott, M. \& Clark, J. R. Distant metastases from cutaneous squamous cell carcinoma--analysis of AJCC stage IV. Head Neck 35, 72-75, (2013).

[21] Song, S. S. et al. Nonmelanoma Skin Cancer With Aggressive Subclinical Extension in Immunosuppressed Patients. JAMA Dermatol 152, 683-690, (2016).

[22] Brougham, N. D., Dennett, E. R., Cameron, R. \& Tan, S. T. The incidence of metastasis from cutaneous squamous cell carcinoma and the impact of its risk factors. J Surg Oncol 106, 811$815,(2012)$.

[23] Hoersch, B., Leiter, U. \& Garbe, C. Is head and neck melanoma a distinct entity? A clinical registry-based comparative study in 5702 patients with melanoma. Br J Dermatol 155, 771-777, (2006).

[24] Martinez, J. C. et al. Defining the clinical course of metastatic skin cancer in organ transplant recipients: a multicenter collaborative study. Arch Dermatol 139, 301-306 (2003).

[25] Ong, C. S., Keogh, A. M., Kossard, S., Macdonald, P. S. \& Spratt, P. M. Skin cancer in Australian heart transplant recipients. J Am Acad Dermatol 40, 27-34 (1999).

[26] Garrett, G. L., Lowenstein, S. E., Singer, J. P., He, S. Y. \& Arron, S. T. Trends of skin cancer mortality after transplantation in the United States: 1987 to 2013. J Am Acad Dermatol 75, 106112, (2016).

[27] Harwood, C. A. et al. A surveillance model for skin cancer in organ transplant recipients: a 22year prospective study in an ethnically diverse population. Am J Transplant 13, 119-129, (2013).

[28] Rashtak, S. et al. Incidence and risk factors for skin cancer following lung transplantation. J Am Acad Dermatol 72, 92-98, (2015).

[29] Jiyad, Z., Olsen, C. M., Burke, M. T., Isbel, N. M. \& Green, A. C. Azathioprine and Risk of Skin Cancer in Organ Transplant Recipients: Systematic Review and Meta-Analysis. Am J Transplant, (2016).

[30] O'Donovan, P. et al. Azathioprine and UVA light generate mutagenic oxidative DNA damage. Science 309, 1871-1874, (2005).

[31] Perrett, C. M. et al. Azathioprine treatment photosensitizes human skin to ultraviolet A radiation. Br J Dermatol 159, 198-204, (2008).

[32] Sugie, N., Fujii, N. \& Danno, K. Cyclosporin-A suppresses p53-dependent repair DNA synthesis and apoptosis following ultraviolet-B irradiation. Photodermatol Photoimmunol Photomed 18, 163-168 (2002).

[33] Dantal, J. et al. Effect of long-term immunosuppression in kidney-graft recipients on cancer incidence: randomised comparison of two cyclosporin regimens. Lancet 351, 623-628, (1998).

[34] Marcen, R. et al. Influence of immunosuppression on the prevalence of cancer after kidney transplantation. Transplant Proc 35, 1714-1716 (2003).

[35] Glover, M. T., Deeks, J. J., Raftery, M. J., Cunningham, J. \& Leigh, I. M. Immunosuppression and risk of non-melanoma skin cancer in renal transplant recipients. Lancet 349, 398, (1997).

[36] Otley, C. C. \& Maragh, S. L. Reduction of immunosuppression for transplant-associated skin cancer: rationale and evidence of efficacy. Dermatol Surg 31, 163-168 (2005).

[37] Epstein, E., Epstein, N. N., Bragg, K. \& Linden, G. Metastases from squamous cell carcinomas of the skin. Arch Dermatol 97, 245-251 (1968).

[38] Alberu, J. et al. Lower malignancy rates in renal allograft recipients converted to sirolimusbased, calcineurin inhibitor-free immunotherapy: 24-month results from the CONVERT trial. Transplantation 92, 303-310, (2011). 
[39] Alter, M. et al. Non-melanoma skin cancer is reduced after switch of immunosuppression to mTOR-inhibitors in organ transplant recipients. J Dtsch Dermatol Ges 12, 480-488, (2014).

[40] Gu, Y. H., Du, J. X. \& Ma, M. L. Sirolimus and non-melanoma skin cancer prevention after kidney transplantation: a meta-analysis. Asian Pac J Cancer Prev 13, 4335-4339 (2012).

[41] Lipson, E. J., Bodell, M. A., Kraus, E. S. \& Sharfman, W. H. Successful administration of ipilimumab to two kidney transplantation patients with metastatic melanoma. J Clin Oncol 32, e69-71, (2014).

[42] Qin, R., Salama AKS. Report of ipilimumab in a heart transplant patient with metastatic melanoma on tacrolimus. Melanoma Manage 2, 311-314 (2015).

Citation: Muneeb Ilyas and Amit Sharma. Clinical Findings, Treatments and Outcomes of Transplant Recipients with Metastatic Skin Cancer. ARC Journal of Dermatology. 2017;2(1):1-10.

Copyright: $\odot 2017$ Muneeb Ilyas and Amit Sharma. This is an open-access article distributed under the terms of the Creative Commons Attribution License, which permits unrestricted use, distribution, and reproduction in any medium, provided the original author and source are credited. 